\title{
XXXII. A quantitative determination of the anomalous dispersion of sodium vapour in the visible and ultra-violet regions
}

\section{R.W. Wood}

To cite this article: R.W. Wood (1904) XXXII. A quantitative determination of the anomalous dispersion of sodium vapour in the visible and ultra-violet regions, Philosophical Magazine Series 6, 8:45, 293-324, DOI: 10.1080/14786440409463201

To link to this article: http://dx.doi.org/10.1080/14786440409463201

曲 Published online: 15 Apr 2009.

Submit your article to this journal $[\pi$

Џll Article views: 8

Q View related articles $\longleftarrow$

Citing articles: 8 View citing articles $\square$ 
LONDON, EDINBURGH, AND DUBLIN

\title{
PHILOSOPHICAL MAGAZINE
}

\author{
AND \\ JOURNAL OF SCIENCE.
}

[SIXTH SERIES.]

$S E P T E M B E R \quad 1904$.

XXXII. A Quantitative Determination of the Anomalous Dispersion of Sodium Vapour in the Visible and Ultra-violet Regions. By R. W. Woon, Professor of Experimental Physics, Johns Hopkins University*.

[Plates X.-XII.]

TTHE dispersion of a medium with but a single band of 1 metallic absorption is represented by the formula

$$
n^{2}=1+\frac{m \lambda^{2}}{\lambda^{2}-\lambda_{m}^{2}}
$$

in which $\lambda_{m}$ is the wave-length at the centre of the absorptionband, and $m$ a constant. This formula, which is developed from electromagnetic considerations, is practically identical with the original Sellmeier formula. Up to the present time it has never been tested experimentally for the reason that no medium of suitable constitution is known which admits of accurate determinations of the variation of the refractive index with the wave-length. We possess such a medium in the vapour of metallic sodium, but the experimental difficulties which stand in our way are so great, that only qualitative observations have been made up to the present time.

The formula given above, being the simplest dispersion formula which we possess, ought by all means to be tested experimentally; and it was with this end in view that the work reported in the present paper was undertaken.

After a large amount of fruitless work, methods have at

* Communicated by the Author.

Phil. Mag. S. 6. Vol. 8. No. 45. Sept. 1904. 
294 Prof. R. W. Wood on the Dispersion of Sodium

length been developed which have made possible accurate determinations of the absolute refractive index of the metallic vapour at varions densities, for all wave-lengths comprised between the extreme red and the remote ultra-violet. For wave-lengths immediately adjoining the $\mathrm{D}$ lines the refractive index has been found to have a value as high as $1 \cdot 38$, as great as that of some liquids, while the dispersion is so great even at the position of the D line of helium, that, could we form a prism of the vapour giving the same deviation as a $60^{\circ}$ glass prism, we could by its aid separate a close Zeeman doublet by an amount as great as the distance between the red and blue of the spectrum formed by the glass prism. The results obtained have been applied to the formula, and most excellent agreement found.

The anomalous dispersion of the vapour was first observed by Kundt, and has since been studied by Becquerel, Julius, Ebert, and others. These investigators have for the most part limited their observations to the dispersion produced by flames of prismatic form, containing the vapour of sodium. Under these conditions the anomalous dispersion is only apparent in the immediate vicinity of the $\mathrm{D}$ lines, extending to a distance up and down the spectrum not much greater than the distance which separates the lines. Some four years ago it was shown by the writer * that by employing the vapour of the metal in glass tubes, it was possible to obtain far greater deviations, and to extend the observations on the dispersion from the extrene red to the violet. These results were made possible by the very remarkable physical properties of the vapour, which will be discussed more in detail presently. The prisms formed in heated glass tubes are in reality nonhomogeneous cylinders of the vapour, the density being greatest along the heated floor of the tube and least along the top. The definition given by non-homogeneous masses of vapour is surprisingly good, and relative values of the refractive indices can be determined without difficulty; but as no means could be found of determining the angle of the equivalent prism, it was impossible to do more than guess at the absolute values. An attempt made several years ago to obtain data by interference methods gave no results, owing to the fact that the fringes were immediately obliterated by the convection-currents of heated hydrogen. By adopting the expedient of heating the metal in a high vacuum, it has been found possible to obtain deviations as high as 1500 fringewidths with the $\mathrm{D}_{3}$ light of helium, without in any way

* Wood, Proc. Roy. Soc. (1901) \& Phil. Mag. [6] vol. iii., "The Anomalous Dispersion of Sodium Tapour." 
altering the appearance of the fringes. By combining the data obtained with the interferometer with the relative values obtained by prismatic deviation, it has been possible to obtain absolute values for a very wide range of wave-lengths. We will begin with a brief discussion of the properties of the medium.

\section{Physical and Optical Properties of the Vapour of Metallic Sodium.}

One of the chief difficulties which has stood in the way of measuring the optical constants of sodium vapour is the impossibility of confining the vapour in vessels bounded by flat surfaces of glass. As soon as the temperature is raised to such a point that vapour of any considerable density forms, the glass is attacked and rendered opaque by the reduction of silica. The vapour, however, has a most remarkable viscosity, which I am at the present time investigating, which makes it possible to form a mass of great density separated from the glass plates which close the ends of the tube by a high vacuum. If we place a number of pieces of clean sodium in a tube of hard Jena glass, the ends of which are closed with small pieces of thin plate-glass, and exhaust the tube on a mercurial pump, on heating the under side strongly with small Bunsen flames, the sodium vapour shows very little inclination to distil to the cold parts of the tube. It condenses to be sure on the upper side of the tube, but is given off so much more rapidly from the surface of the molten metal than it can diffuse to the upper portion, that the density gradient is very steep. Observations on the deviation produced by the non-homogeneous cylinder show that the equivalent prism has a form similar to that shown in fig, 1, the density gradient

Fig. 1.

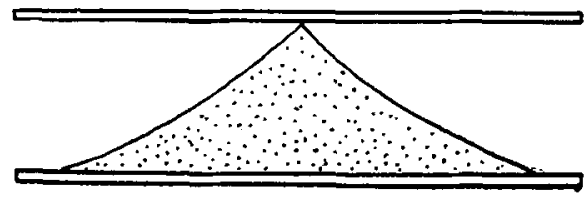

being steeper near the bottom of the tube. To secure good definition it is therefore necessary to place in front of the tube an opaque screen, perforated with a wide horizontal slit. The tube thus prepared, used in the manner to be presently described, shows the strong anomalous dispersion in the vicinity of the $\mathrm{D}$ lines with great distinctness. If the flames are made to play upon the upper surface of the tube, $\mathrm{X} 2$ 
after some of the vapour has condensed there, the spectrum straightens out, and the dispersion presently occurs in the opposite direction, showing that the base of the prism is always against the heated portion of the tube regardless of gravity. In a tube $25 \mathrm{cms}$. long, $10 \mathrm{cms}$. of which are heated red-hot on all sides by means of an iron wire carrying a heavy current, a mass of sodium vapour may form of sufficient density to give a displacement of several thousand helium $\left(D_{3}\right)$ fringes; notwithstanding this, practically no trace of the vapour can bo detected beyond the heated portion. This condition can be maintained for an hour or more, owing to the slowness of the diffusion. If mercury is heated under similar conditions, it is impossible to obtain shifts of more than a fringe or two. Potassium distils almost in a flash to the colder parts of the tube. The other alkali metals have not as yet been investigated. The vapour of sodium appears to possess a cohesion almost as great as that of a liquid. Even in a vacuum-tube it appears to have a free surface, while in an atmosphere of hydrogen it can be dipped up on a wire, a vigorous shaking of which is insufficient to dislodge it or cause its dissipation. A number of very interesting experiments with the vapour have already been made, and others are in progress at the present time. They will be reported in a subsequent paper, as for the present we are only concerned with the fact that a homogeneous cylinder of very dense vapour terminated with sharply defined ends can be formed in an exhausted tube.

Anomalous dispersion occurs at the absorption-lines which belong to the principal series, being very strong at the $D$ lines, feeble at the first pair of ultra-violet lines $(\lambda=3303)$ and almost imperceptible at the second pair $(\lambda=2852)$, as is shown in fig. 2 . If the vapour is of considerable density,

Fig. 2.

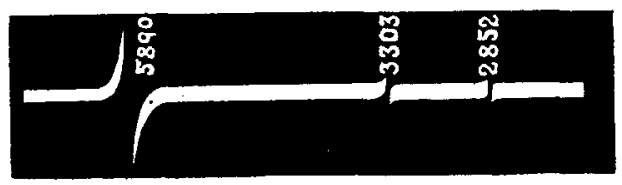

the $\mathrm{D}$ lines run together into a single absorption-band, and the dispersion can be represented throughout the complete range of wave-lengths as resulting from electrons of a single period, for the ultra-violet bands only affect the refractive index in their immediate vicinity. These bands make their appearance in succession as the density of the vapour increases, the second not appearing until the band at the $D$ lines 
has become three or four times as broad as the distance between the lines. The complicated channelled absorption spectrum appears to exert no influence on the refractivity of the vapour. I feel certain, however, that the channelled absorption spectrum affects the refractivity of the medium in a small degree, and intend to test the mattier in the near future by a modification of the method of striæ. The channelled bands affect the magnetic rotatory dispersion in a powerful degree, and this subject is under investigation at the present time. The magnetic dispersion is many times greater than the ordinary dispersion, as has been found by examining the vacuum dispersion-tubes in a powerful magnetic field with polarized light, and the whole subject will be fully taken up in a subsequent paper.

The absolute values of the refractive index for different densities have been determined with the interferometer for monochromatic light of different wave-lengths. Relative values (dispersion) have been obtained in the same way, as well as by the method of crossed prisms.

\section{Determination of the Dispersion with the Interferometer.}

The absolute determinations being the more difficult, it was decided to begin with an investigation of the dispersion, in order to get as much experience as possible with the behaviour of the vapour under various conditions. This was a wise decision, as was subsequently found, for a number of very elusive sources of error were discovered, which were eliminated in the final work.

The general plan of the apparatus is shown in Plate $\mathrm{X}$. The interferometer was the form of the Michelson instrument designed by Brace.

The base of the instrument consisted of a heavy tube of brass $12 \mathrm{cms}$. in diameter and a little over a metre long. The arrangement of the mirrors is shown in the diagram, all but one being of speculum metal. Light from an arc-lamp was. focussed on the slit of the monochromatic illuminator. The beam issuing from the slit of this instrument comprised a range of wave-lengths about equal to the range between the D lines. Near the slit was mounted a helium tube, which furnished a light of constant wave-length, and the observations consisted in counting the fringe-shifts for the two kinds of light when a given amount of sodium vapour was introduced into the path of one of the interfering beams. A small mirror was mounted in front of the interferometer, which deflected a portion of the emergent light into a telescope, the remainder of the beam entering a second telescope, By a 
little adjustment it was possible to get the fringes formed by helium light distinct in one telescope, and those formed by the light from the monochromatic illuminator distinct in the other. The helinm tube standing a little to one side of the slit, very little overlapping of the two systems occurred.

The sodium was contained in a seamless steel tube, covered with asbestos paper and wrapped with a helix of iron wire through which a heavy current was passed. The ends of the tube were closed with plates of glass, which were of such a thickness that they served in place of the compensating plate which is usually placed parallel to the transparept mirror. The tube was exhausted by means of a mercurial pump, with which it was kept in connexion throughout the experiments in order to remove the occluded hydrogen which was given off as soon as the sodium was heated. This occluded gas gave a good deal of trouble, and the behaviour of the fringes seemed to indicate that a small amount of the gas became entangled in the sodium vapour, no amount of pumping appearing to remove it entirely. Its effect on the fringe-shift will be discussed presently.

The $\mathrm{D}_{3}$ line of helium was so strong in the tube used that no colour-screens were necessary, the fringes being as distinct as those obtained with sodium light. Owing to its proximity to the absorption-band, very large fringe-displacements were obtained with a small amount of comparatively rare sodium vapour.

In the electrically-heated tubes the sodium vapour showed no tendency to form the non-homogeneous equivalent of a prism, and the fringes consequently remained straight, even after very great displacements.

Two observers were necessary ; one counting the helium fringes as they passed the cross-hair of the telescope, and the other noting the transit of the fringes formed by the light from the monochromatic illuminator.

If the light used was on the same side of the D lines as the helium line, the two sets of fringes were shifted in the same direction; if on the other side, the shifts were in opposite directions. In addition to comparing light from the monochromatic illuminator with the helium light, a very careful comparison was made with the green mercury line, since this light was to be used in the absolute determinations.

At first very discordant results were obtained, but the source of error was soon discovered to lie in the heating and consequent expansion of the base-bar of the interferometer by the radiation from the sodium tube. The instrument was then 
covered with layers of cotton and asbestos board, and consistent results were at once obtained. There was still a source of trouble which for a long time remained undiscovered. On heating the tube it was found that the relative shifts changed as the temperature rose, becoming constant after a shift of about a dozen helium fringes. Repeated heating of the sodium to a high temperature, combined with pumping, lessened the discrepancies, but the trouble was never completely eliminated. It is now believed that a small amount of hydrogen is held captive by the sodium vapour, condensing with it as the tube cools. When the tube is again heated, this occluded hydrogen is evolved with the first traces of sodium vapour, and modifies its refractivity. If we are working with light on the same side of the $D$ lines as the helium line, for which the refractive index of the vapour is less than unity, the evolution of this gas will oppose the displacement of the fringes, holding both sets back by practically the same amount. Suppose we are working with a wave-length for which the relative shift with respect to helium light is $1: 10$. If we form a sufticient quantity of sodium vapour to shift the helium fringes through 30 fringe-widths, and the other set 3 widths, and an amount of hydrogen sufficient to give a shift of one fringe-width has been set free, it is obvious that our observed ratio will be $2: 29$, or in round numbers $1: 15$. The errors observed were always at the beginning of the series when the tube was heated, and at the end when it was cooled, and were always of such a nature as to be explained perfectly by the hypothesis which $I$ have advanced. On this account we always disregarded the relative values found when the sodium vapour was only present in very small quantities.

Many weeks of work were necessary before all sources of error were eliminated and the best methods of working discovered. As a sample of one of the troubles met and overcome, the following will serve. When working with light from the monochromatic illuminator of a wave-length very near that of the $D$ lines, the fringes disappeared before shifts of more than one or two fringe-widths were observed. It had been our habit to set the mirrors of the instrument in such positions that the centre of the fringe system was seen in the telescopes. The disappearance was finally found to bc due to the dispersion of the vapour, and by turning the screw of the instrument in such a way as to increase the length of the air path, they appeared again *. The most

* This action of the vapour on the fringes will be discussed in the paper following the present one (p. 324). 
advantageous way to work was therefore to begin the observations with the maximum air path consistent with visibility of the fringes ; as the sodium vapour formed they increased in distinctness at first and then gradually faded away. In this way it was possible to get a shift twice as great as before; if it was desirable to work with a denser vapour to eliminate the error due to evolved hydrogen, we had only to increase the length of the air path still further, and work further out in the system. Under these conditions the fringes are invisible at the beginning and end of the experiment, only coming into view when a considerable quantity of sodium vapour is present. The same thing is true to a less extent with helium light, the fringes disappearing after a shift of about 200 fringe-widths has taken place. By increasing the length of the air path, either by turning the screw of the instrument, or introducing one or more thick plates of plane-parallel glass, they again come into view, and the counting can be continued. The latter method was preferred, as the glass plate could be dropped into place in a moment without interrupting the count. This trick was only necessary in making the absolute determinations with belium light, as in the comparison work the vapour never attained such a density as to cause the helium fringes to disappear. When working close to the helium line with the monochromatic illuminator it was necessary to work with very narrow slits, otherwise the enormous dispersion of the vapour obliterated the fringes almost immediately, in spite of all precautions. It was impossible to approach closer to the absorption-band than the helium line, owing to this trouble, and ratios of $1: 2$ are about as large as we feel much confidence in, though on one or two occasions we set the monochromatic illuminator on the $\mathrm{D}_{3}$ line, and obtained equal shifts in the two systems. It was impossible to observe a shift of more than one or two fringes in this case before the system disappeared. The most accurate determinations are doubtless those where the ratio is between $1: 3$ and $1: 15$. In the blue-green region the ratio is in the neighbourhood of $1: 40$, and the values are not as accurate, as an accidental displacement of half a fringe-width, which may easily occur as the result of a slight change in the temperature of the instrument or the air, exercises a much greater effect on the results. On this account the determinations made by the prismatic method are to be considered mora reliable in this region of the spectrum. 
Vapour in the Visible and Ultra-violet Regions.

Deternination of the Dispersion in the immediate vicinity of the $D$ Lines.

The extraordinarily high value of the dispersion close to the absorption-band makes it impossible to sift out from the spectrum, light sufficiently homogeneous to yield interferencefringes when even a very small amount of sodium vapour is introduced into the path of the light. It was found difficult to get satisfactory values with the light from the monochromatic illuminator much closer to $\mathrm{D}_{3}$ than the distance separating $\mathrm{D}_{3}$ from $\mathrm{D}_{2}$, the fringes becoming blurred as soon as the smallest trace of sodium vapour was formed. A different method was accordingly adopted which yielded excellent results.

It is clear that if we employ light of two different wavelengths in illuminating the interferometer, the introduction of the metallic vapour will shift the two fringe systems by different amounts, the fringes appearing and disappearing as they get into and out of step in succession. If both systems are shifted in the same direction, which is the case when both wave-lengths are on the same side of the absorption-band, we can measure the dispersion by counting the number of fringes which pass the cross-hair of the telescope between two successive disappearances. If, for example, the number is found to be 15 , we know that one set of waves is retarded or accelerated 15 wave-lengths, under the same conditions which canse a shift of 16 fringes for the other waves. All uncertainty as to the movement of the fringes during a period of minimum visibility was avoided in the method employed, as will appear presently.

To determine the dispersion close to the $\mathrm{D}$ lines we require highly homogeneous light of two different wave-lengths, which would yield a close double line at the point at which we wish to measure the dispersion.

This was accomplished by breaking up the radiation of a helium tube into a Zeeman triplet and cutting out the middle component by means of a Nicol prism placed with its plane of vibration perpendicular to the lines of magnetic force. A large Ruhmkorff magnet with an ammeter in circuit was employed, the key for making and breaking the circuit being placed close to the observing telescope of the interferometer. The distance between the components of the double line obtained when a current of 30 amperes was flowing through the magnet was found to be $1 / 23$ of the distance between the sodium lines. This was determined very easily by counting the number of turns of the interferometer-screw necessary to cause the fringes to pass through a given number 


\section{Prof. R. W. Wood on the Thispersion of Sodium}

of phases of maximum visibility, the method being the one commonly employed in the laboratory in the determination of the relative wave-lengths of the sodium lines. The magnetic field was now thrown off, and the temperature of the heating coil gradually raised. As the sodium vapour formed, the fringes were counted as they drifted across the field. The current was thrown into the magnet at frequent intervals for a fraction of a second, and the fringes were seen to pass through successive phases of visibility and invisibility. The momentary disappearance caused by the magnetic field did not interfere with the counting, for the fringes moved slowly and with the regularity of clockwork. The exact moment at which the fringes disappeared could not of course be accurately determined; since they remained invisible during a period occupied by a shifting of several fringe-widths, but by observing six or seven successive disappearances values were obtained which did not differ by more than two or three per cent. The average of a number of readings showed that the sodium vapour shifted the two sets of fringes in the ratio of 10.5 to 11 , that is fringes formed by waves of length 587487 are shifted 10.5 fringe-widths, while those formed by waves of length 587513 are shifted 11 fringe-widths. These numbers do not look very formidable until we translate them into prismatic deviations. A prism having the same dispersion giving a total deviation of only 11 degrees would separate the two components of the Zeeman triplet half a degree, or if we could construct a sodium-vapour prism giving the same deviation as a $60^{\circ}$ glass prism, two lines in the spectrum twenty-three times as close together as the D lines would appear separated by a distance greater than the distance between the red and the bluish green of the spectrum formed by the glass prism:

The same method was applied to a determination of the dispersion between the yellow mercury lines, for the purpose of obtaining a check on the curve obtained by comparing the shifts of the helium fringes with fringes formed by the light from the monochromatic illuminator.

A prism was placed in front of the observing telescope to separate the yellow fringes from the more intense green ones. On setting them in motion by forming sodium vapour in the path of the light, they were found to disappear periodically in the same manner. It was impossible to get as accurate data as were obtained with the Zeeman lines, since there were moments of complete invisibility, but by counting steadily it was possible to tide over the few seconds when the field was a blank and get a fair estimate of the relative shifts. 
A number of observations were made, the mean of which gave $5: 6$ as the ratio of the shifts of wave-lengths 5770 and 5790 . These values fitted almost exactly on the dispersion-curve obtained with the monochromatic illuminator and the helium tube. Obviously a more accurate method would have been to provide a device by which the light of one of the yellow lines could have been cut off as desired. An attempt was made to do this with the monochromatic illuminator, but the loss of light was too great.

Some very curious results were obtained by illuminating the interferometer with sodium light. The fringes disappeared at the moment when the thermo-element indicated a temperature of $180^{\circ}$. It was at first thought that the disappearance was due to absorption of the light by the vapour in one of the two paths, but on covering up the other path it was found that the field of the instrument was still brightly illuminated. On turning the screw of the instrument the fringes appeared again, and a little further experimenting showed that the effect of the vapour was to alter the visibility curve, its periodicity becoming less as the density of the vapour increased. No satisfactory explanation of the altered appearance of the fringe system could be found, and an attempt was made to get rid of one of the D lines in the illuminating light, thus simplifying the conditions. Prisms and gratings were tried, but the loss of light was so great, that even with an oxyhydrogen sodium flame the fringes could be made out only with the greatest difficulty. The desired result was finally obtained by mounting a quartz crystal between two crossed nicols. A number of erystals were examined with polarized light perpendicular to the axis, and one selected which showed dark bands in the spectrum separated by a distance about double the distance between the $\mathrm{D}$ lines. The nicols were arranged so that the emergent light vibrated in such a plane as to be most copiously reflected by the interferometer mirror, $i . e$. the first nicol was mounted with its short diagonal horizontal, while the one next to the instrument was placed with its iong diagonal horizontal. Between the nicols was mounted the quartz crystal with its axis making an angle of 45 degrees with the vertical. The light from an oxy-hydrogen sodium flame, after passage through the polarizing system, was brought to a focus on a cardboard screen by means of a lens, forming a series of bright bands separated by dark intervals. A narrow slit in the screen allowed light corresponding to $D_{1}$ or $D_{2}$ to enter the interferometer, according as it was set on one edge or the other of one of the bright bands. This arrangement worked admirably, and yielded an abundance of light. No 
trace of the periodic invisibility was found in the fringe system, such as is always observed when ordinary sodium light is used. As soon as the smallest trace of sodium vapour was formed in the tube, the fringes were found to have become quite invisible in certain parts of the system. The visibility curve changed rapidly as the density of the vapour increased, the period becoming smaller. The general behaviour of the fringes did not differ materially from that observed when both sodium lines were used, proving that the presence of the two wave-lengths was not essential. The appearance of fringes under the conditions of the experiment depends upon the fact that the emission-line is broader than the absorption-line. The absorption is restricted to the centre of the line, the edge light only getting through the sodium tube and forming fringes. This edge light is most powerfully dispersed by the vapour, and the two fringe systems which it forms are shifted in opposite directions. It was hoped that a method might be worked out for determining the dispersion of the vapour in the immediate vicinity of the D lines, by studying the behaviour of sodium light when dispersed in the interferometer, and a great deal of time was lost in the endeavour to unravel the very complicated changes produced in the appearance of the fringe system. If we were dealing with strictly homogeneous radiations, lying to the right and left respectively of the absorption-band, the two fringe systems would move in opposite directions at the same rate, and we should have simply rapid alternations of visibility and invisibility. By the simultaneous use of a helium tube we could determine the number of disappearances of the sodium fringes corresponding to a shift of one or more helium fringes, which would give us at once a measure of the shift of the system formed by the sodium light. This would give us a measure of the refractive index closer to the absorption-bands than we could get by any other method. An attempt was made to utilize the Zeeman phenomenon in this case, the sodium flame being placed in a magnetic field and the interferometer illuminated with one or the other of the D lines, by means of the polarizing system. There was no difficulty in finding positions of the interferometer mirror for which the fringes were visible with the field on, and invisible when it was off ; but nothing resembling a rapid appearance and disappearance of the fringes was observed when the sodium vapour was formed. This is probably due to a number of causes. In the first place, the light from the flame is by no means strictly homogeneous, for a pale flame, containing only a little sodium and yielding narrow lines, 
does not give light enough. In the second place, as is well known, the $\mathrm{D}$ lines do not break up into polarized triplets in the magnetic field, but into a quadruplet and sextuplet. At the time of trying the experiment this circumstance was not thought of, and the failure may have been due in part to the fact that only $\mathrm{D}_{2}$ was utilized, which is obviously less suited to the purpose, for the reason that it gives us a double line on each side of the absorption-band when the central components are cut out by the nicol. I am of the opinion, however, that the finite breadth of the normal line is chiefly responsible for the failure. Some experiments were made with vacuumtubes containing sodium vapour, which yield highly homogeneous light, but they were found troublesome to manage. It is quite possible that by the use of these tubes, results could be obtained in the case of $D_{1}$, and utilizing the light emitted in the direction of the lines of force (Zeeman doublet) we might get data for both lines. This experiment would have been tried were it not for the fact that almost as satisfactory results can be obtained by the method of prismatic deviation, with a much less expenditure of time.

As a discussion of the modifications of the appearance of the fringes formed by sodium light, by the introduction of the vapour of sodium into the path of one of the interfering beams of light, has no especial bearing upon the determination of the dispersion of the vapour, it will be postponed for the present. A fuller discussion will be given in a subsequent paper on "The Achromatization of Fringes formed by approximately homogeneous light by highly dispersing media."

\section{Prismatic Deternination of the Dispersion in the immediate vicinity of the $D$ Lines.}

As I have already shown, prisms of excellent defining power can be formed by heating metallic sodium in highly-exhausted tubes of hard glass. In my earlier experiments, in which the metal was beated in an atmusphere of hydrogen, the definition was not good enough to obtain a smooth curve close to and between the $D$ lines by the method of crossed prisms. The photographs illustrating my first paper, in which the dispersion between the lines was shown, were taken by employing a prismatic flame of hydrogen burned at a platinum jet, and fed with the vapour of sodium. By using vacnum-tubes heated by very small flames far better results can be secured, and perfectly smooth sharp curves obtained. Two different methods were used in the determination of the deviation produced by the prism for wave-lengths close to the $D$ lines. 
306 Prof. R. W. Wood on the Dispersion of Sodium

A reticulated mesh was ruled on a small plate of glass with one of the Rowland dividing-engines. This plate was mounted in the eyepiece of the spectroscope on the slit of which the image of a horizontal slit was thrown, after dispersion by the sodium tube. The spectroscope was provided with a plane grating ruled with 20,000 lines to the inch, which gave a very brilliant 2 nd order spectrum. There were four squares of the reticulated mesh between the $\mathrm{D}$ lines, and nine squares between $D_{2}$ and $D_{3}$.

As the temperature of the sodium tube was raised, the relative deviations in the different squares of the mesh were noted. The light immediately adjacent to the $\mathrm{D}$ lines was deviated entirely out of the field of the telescope before any measurable deviation at the helium line occurred. At a temperature at which the spectrum at the helium line was deviated the width of one square, the wave-lengths between the $\mathrm{D}$ lines, and for a distance of two squares to each side of them, were lost by absorption. By means of step by step measurements, taken at different temperatures, it was possible to get a very good idea of the dispersion curve within this very

Fig. 8.

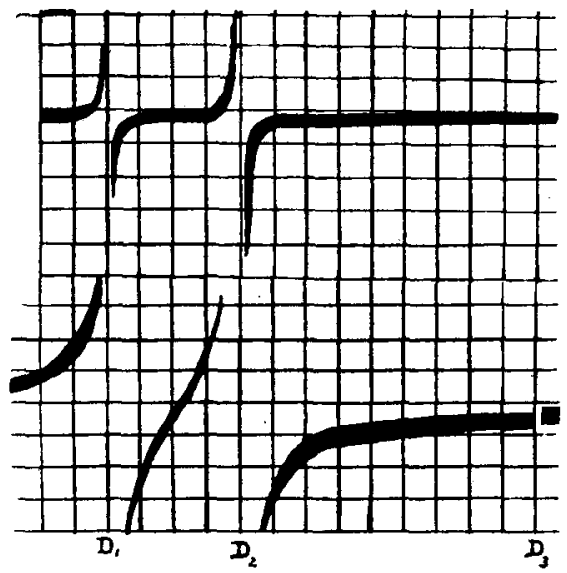

narrow range of the spectrum. The appearance of the spectrum on the reticulated background at two different temperatures is shown in fig. 3 , the dispersion at $D_{2}$ being somewhat greater than at $D_{1}$. Observations were taken of the dispersion within a quarter of a square of $\mathrm{D}_{2}$, that is within a distance of the absorption-line equal to $1 / 16$ of the distance between the $\mathrm{D}$ lines. The deviation at this point was calculated to be about 
80 times the deviation at the helium line. If we number the wave-lengths at the lines of the mesh between $D_{3}$ and $D_{2} 1$ to 9, the observations were as follows:-The light very close to $D_{2}(1 / 4$ of a square distant) was seen to be distinctly pulled down through a distance of seven squares with a vapour-density sufficient to deviate the spectrum at the first line $(=5888 \cdot 3)$ through a distance of one square. The temperature was then raised a trifle. The light very close to $D_{2}$ now disappeared, partly as a result of absorption and partly through being deviated out of the field. The spectrum at the first line was deviated through three squares, that at the second line one square. The temperature was now raised until the spectrum at the 9th line was deviated through one square. The deviation at the 2 nd was now four, that at the $3 \mathrm{rd}$ three squares, and that at the 5th two squares.

These results for the different wave-lengths between $D_{3}$ and $D_{2}$ are given in the following table:-

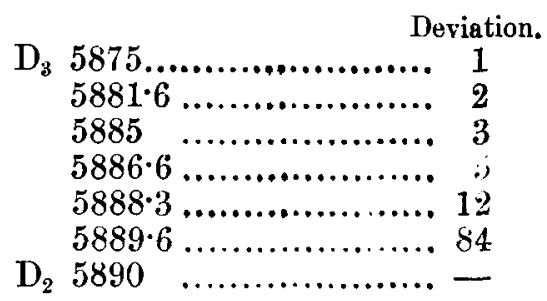

These values are of course only approximations, the errors becoming greater as we approach $\mathrm{D}_{2}$. The same experiment was repeated, using a filar micrometer in the eyepiece in place of the reticulated mesh, and values obtained which agreed fairly well with those given above. This method, however, did not admit of such a near approach to $D_{2}$ as the other.

From these results it is possible to calculate the values of the refractive index within this range for the very dense vapour used in the absolute determinations. These will be called observed values, and they will be compared with the values calculated from the dispersion formula for the corresponding wave-lengths.

I have already spoken of the enormous dispersion at the helium line, a dispersion great enough to separate a Zeeman doublet by an amount equal to nearly the width of the entire visible spectrum produced by a glass prism of $60^{\circ}$, provided we were able to construct a prism of sodium vapour giving: the same mean deviation, and get the light in question through it. Great as is this dispersion it is as nothing when compared 
with that within say an Ångstrom unit of one of the D lines, as an inspection of the above table indicates.

The dispersion within the range between $\mathrm{D}_{2}$ and $\mathrm{D}_{3}$ is shown graphically in Curve 1, Pl. XI., ordinates and abscissæ being inside the small rectangle.

If this curve were completed up to $\lambda=58896$, at which the largest recorded deviation occurs, the point in question would be at the 76 th square below the unity line. To include this point it would have been necessary to take the ordinates of the curve on about $1 / 4$ of the scale employed.

Visual Observations of the Dispersion in the Visible Spectrum by the Method of Crossed Prisms.

As a check on the results obtained with the interferometer, a series of measurements of the dispersion was made by the method of crossed prisms. The method was identical with that described in my previous paper, except that the metal was heated in a highly exhausted tube of hard Jena glass instead of in a tube filled with hydrogen. These tubes can be very easily prepared, thd give "no trouble at all. They are, however, apt to crack if reheated, after having been allowed to cool. It is not necessary to have the sodium in separate small lumps, as the action is not the formation of a number of prisms of sodinm 'vapour, but 'a' eylinder of vapour, very dense along the heated floor of the tube, and decreasing to very nearly zero along the top. If it is desired to prepare a tube for purposes of demonstration, a tube of thin steel is preferable, as it can be used over and over again. In this case it is necessary to lay a thick pad of wet asbestos-paper along the top of the tube, since the more uniform heating which results from the better conductivity of the steel is detrimental to the formation of the non-homogeneous cylinder of vapour, which only takes place when the temperature gradient between the top and bottom of the tube is very steep. The plate-glass ends are fastened on with sealing-wax, a small glass tube being sealed in in the manner described in my previous paper, through which the air is exhausted. (See fig. 4.)

Fig. 4.

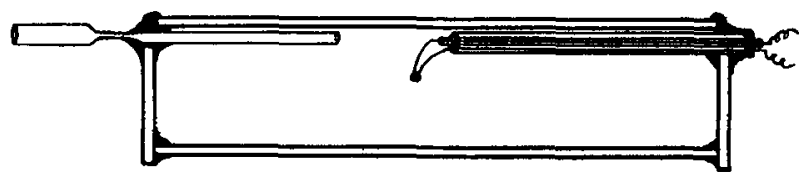

Glass tubes were used in making the measurements, as there was no occasion for cooling the tube and reheating it, 
and the temperature gradient takes care of itself in this case. The sodium was first melted in a small iron crucible and poured into an improvised sodium press; a piece of apparatus so easily made and so useful that a brief description of it may not be out of place. A steel cylinder is bored with a hole two oi three cms. in diameter to within a few millimetres of its end. A 3-mm. hole is then drilled through the end at the centre of the larger hole. A solid steel piston is turned so as to fit easily into the bore of the larger cylinder. The molten sodium is poured in, allowed to cool, and the piston inserted. The whole is then put into the jaws of a large vice, and the sodium wire squeezed out, directly into the dispersion-tube. In this way the formation of oxide is prevented, and the sodium is freed from the hydrocarbon in which it is kept. The glass plate is immediately cemented on, and the tube exhausted on a mercurial pump. An ordinary hand-pump would doubtless answer every purpose, as the vacuum does not need to be below a few millimetres. I see no reason why a well-made steel tube should not be used year after year in class demonstration, though I have not tried keeping one so long. With the tube once prepared, the apparatus for showing the anomalous dispersion of the vapour, which is one of the most beautiful optical experiments that I know of, can be set up in five minutes. The former method which I described was rather troublesome, as it involved the use of a hydrogen generator and drying-tubes, and it was often difficult to avoid the formation of smoke in the tube. The vacuum-tubes make the experiment as easy to perform as the simplest of the laboratory demonstrations. A combustion furnace is not necessary, two or three Bunsen burners turned down low answering every purpose. The amount of dispersion can be regulated by turning the flames up or down. A full description of the disposition of the apparatus will be found in my first paper. In brief, it consists of a horizontal slit on which the crater of an arc-lamp is focussed. If a Nernst lamp is available it may advantageously be substituted for the illuminated slit, the glower being mounted in a horizontal position. The light from the slit is made parallel with a lens, and after traversing the sodium tube is focussed on the slit of a small spectroscope by means of a second lens. It is almost as satisfactory to use a single lens passing the convergent beam through the dispersion-tube.

If it is desired to show the anomalous spectrum, a lowpower eyepiece is substituted for the spectroscope. The appearance of this spectrum is shown by a coloured plate illustrating the earlier paper. In the present work it was desirable Phil. Mrg. S. 6. Vol. 8. No. 45. Sept. 1904. Y 
to have a normal spectrum, and the spectroscope was replaced by a collimator and plane grating. The light from the grating entered a camera made of a long wooden box, provided with an achromatic telescope-leris of 2 metres focus.

The spectrum, which was received on a plate of ground glass, was about $25 \mathrm{cms}$. long and $1 \mathrm{~mm}$. wide. Its position was accurately recorded on the ground glass by means of a narrow pencil mark ruled along its centre, the ground surface having been sponged over with oil to increase its transparency. The burners of the furnace were now lighted and turned down very low so that the tips of the flames fell a little short of the tube. As soon as the temperature of the tube became constant, the curved branches of the dispersed spectrum were outlined on the glass with the pencil. The burners were then turned up a little and the deviations of the oppositely curved branches increased, their new positions being recorded in the same manner. A number of records were made in this way, and the tube was then allowed to cool off. The spectrum straightened, and was found to occupy the same position on the plate as before, showing that no displacement of the apparatus had occurred. A cadmium spark and sodium flame were now placed before the slit of the grating spectroscope, and the position of the bright lines recorded on the plate. The deviations which different regions of the spectrum had experienced were then carefully measured. The object of making a number of records was to show whether the relative deviations depended on the temperature of the tube and density of the vapour. No indications of any such irregularity were found. The values found in this way agreed admirably with the results obtained with the interferometer. It moreover furnished data regarding the dispersion in the blue and violet region, which the interferometer failed to do with any accuracy for reasons which I have given. Photographs of the dispersed spectrum are reproduced on fig. 8, Plate XII. They are neither retouched nor enlarged, and were made with sunlight; the channelled absorption spectra in the red and green appear on the plate.

\section{Numerical Results.}

The results obtained by the three methods are given in the second column of the table in the section deroted to the refraction and dispersion of sodium vapour of great density, which follows. For the sake of uniformity they have all been reduced to the same scale. A shift of 100 belium $\left(D_{3}\right)$ 
fringes being taken as the standard. A wave-length, for which the relative shift with respect to helium light of the interferometer-fringes was found to be $1: 4$, is entered in the table as 25 .

The shift ratio for the green line of mercury with respect to helium was found to be $1: 25$. The wave-length of this light is, therefore, represented in the table by the numeral 4, which means that a prism of sodium vapour which gives a deviation 4 for green $H_{g}$ light gives a deviation 100 for helium light. The results obtained by the methods of crossed prisms (both visual and photographic) were reduced to the same scale, being compared with the value found for the green mercury line. This was necessary, since if a vapour of sufficient density is used to give a measurable deviation in the blue and violet region, it absorbs almost entirely that portion of the spectrum occupied by the helium line. The values obtained by means of the Zeeman doublet and the two yellow lines of mercury, by the method of coincidences, are not given in the tablo. As these values were purely relative, i. e. not compared with helium or any other standard wave-length, it was necessary to assign to one of the wavelengths a shift taken from the curve determined by the other methods, and then calculate the shift of the other wave-length. For example, in the case of the yellow mercury lines, of wave-lengths 577 and 579 , the ratio as obtained by the coincidence method was $5: 6$. The shift of 577 on the curve was found to be $14, i$.e. 14 of these fringes to 100 of helium 5 . The shift of wave-length 579 with respect to helium is given by the proportion $5: 6=14: x$, from which we get $x=16 \cdot 8$, a value which fell exactly on the curve obtained with the interferometer.

The same thing was done in the case of the values obtained by breaking the helium line up into a Zeeman doublet, 11 fringes for $\lambda=587487,11.5$ fringes for $\lambda=587513$. The values for the range of wave-lengths comprised between the helium line and $\mathrm{D}_{2}$ were determined by the method of crossed prisms, the measurements being made with the reticulated mesh in the focus of the eyepiece. For light of wave-length midway between $D_{3}$ and $D_{2}$ the deviation is twice as great as for $D_{3}$, consequently we enter this in the table as 200 .

\section{The Ultra-violet Dispersion.}

A qualitative study of the dispersion in the ultra-violet was first made by the method of crossed prisms.

A tube of hard Jena glass, $4 \mathrm{~J} \mathrm{cms}$. long and $1.5 \mathrm{cms}$. $\mathrm{Y} 2$ 
internal diameter, was fitted with end-plates of quartz, loaded with sodium and exhausted. The arrangement of the apparatus is shown in fig. 5. As the method depends on forming a sharp image of a brightly illuminated horizontal slit upon the vertical slit of the spectrograph, quartz lenses could not be used owing to their chromatic aberration. Concave silvered mirrors are free from this defect, but have abnormally low reflecting power in the ultra-violet region. Very satisfactory photographs were obtained with them, however, in the preliminary work, though they were replaced by magnalium reflectors later on. The light from the crater of an arc-lamp was focussed on the horizontal slit, and collimated with one of the concave mirrors. It then passed through the sodium tube, after which it was brought to a focus by a second mirror on the slit of a quartz specFig. 5.

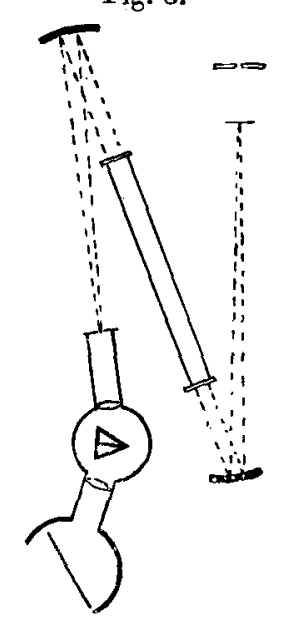
trograph. The tube was heated by a row of small Bunsen burners, and as soon as a steady state was reached, which could be told by observing the curved branches of the spectrum with an eyepiece, the photographic plate was inserted and exposed. Owing to the low reflecting power of the silver the ultraviolet region was somewhat underexposed. The curvature of the spectrum in the immediate vicinity of the first two ultra-violet lines of the principal series was very pronounced, however. The plates obtained with the small quartz spectrograph are too small to reproduce well, especially as the density is not very great in the most interesting region, and it has seemed better on this account to make drawings from them. The general appearance of the photographed spectrum is shown in fig. 2 (p. 296), which has been enlarged about two diameters. It is apparent that the effect of these lines on the refractivity of the medium is negligible except in their immediate vicinity.

Plates obtained in the manner described were useless for purposes of measurement, as no record was made of the position of the spectrum on the plate before its deviation by the sodium prism.

As the displacement is very slight in the remote ultraviolet the two records would overlap in this region, even if the spectrum was very narrow. To overcome this difficulty the following method was devised. A slide was fitted to the 
plate-holder, perforated with a number of vertical slits, each about $2 \mathrm{mms}$. wide, the widths of the opaque bars and slits being equal. This slide was left in the plate-holder during the exposures, and screened off the spectrum at regular intervals. In order to obtain greater deviations a large tube of Jena glass $80 \mathrm{cms}$. long was used. It was heated in z. combustion furnace, the burners of which were turned down low, the best results being obtained when the tops of the flames only partially enveloped the tube.

As it was desired to extend the observations as far down into the ultra-violet as possible, the spark was substituted for the arc, and to avoid loss of light by reflexion one of the mirrors was dispensed with. The spark was furnished by a large induction-coil operated with an alternating current of 110 volts, a leyden-jar of large size having been joined in parallel with the terminals. Cadmium electrodes were used, the spark-gap being placed close to the horizontal slit, whieh was mounted at the centre of curvature of a concave reflector of magnalium of 1 metre radius. The convergent beam from the reflector passed through the sodium dispersion-tube and came to a focus on the slit of the spectrograph. The bottom of the tube having been raised to a full red heat in the combustion-furnace, the photographic plate was exposed with the slotted slide in position. The anomalously dispersed spectrum was thus recorded in small sections corresponding to the slits in the slide. The tube was then allowed to cool off, the slide drawn out a distance equal to the width of one of the slits,

Fig. 6.

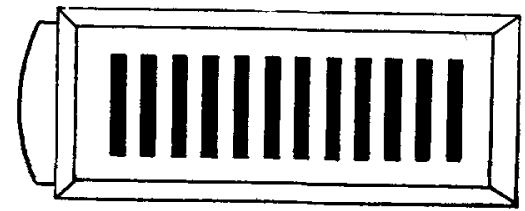

Fig. T.

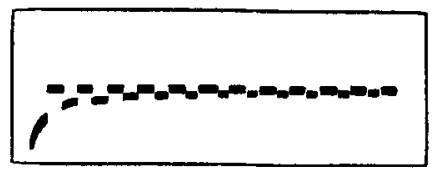

and a second exposure made which recorded the position of the undeviated spectrum. A drawing of the plate-holder, with its perforated slide and the deviated and undeviated spectra obtained with it, are shown in figs. $6 \& 7$. 


\section{Prof. R. W. Wood on the Dispersion of Sodium}

Absolute Determinations of the Refractive Index.

The deviations of the light-rays by the non-homogeneous cylinders of sodium vapour formed in exhausted tubes gives us no measure of the absolute refractive index, for the reason that we have no way of determining the angle of the equivalent prism. Various methods were tried designed to give to a mass of vapour of uniform density a prismatic form. None of them was very satisfactory, and until the discovery was made that a fairly uniform cylinder of vapour could be formed in an exhausted tube, the absolute value of the index for any given wave-length could only be guessed at.

The results found for the dispersion with the interferometer showed that results correct to within probably 5 per cent. could in all probability be obtained by this method.

The density of sodium vapour at different temperatures has been investigated by Jewett (Phil. Mag. iv. p. 551, 1902) for a range comprised between $368^{\circ}$ and $420^{\circ}$. It was accordingly determined to take the temperature of the vapour as a measure of its density.

A thermo-couple of iron and constantan wire was made by holding the ends of the wires together, both being connected to a storage-battery giving an E.M.F. of about 20 volts. The other wire of the battery was then touched momentarily to the tips of the two wires, the small are which formed on separation fusing them together very neatly. I do not remember to have seen this method of making thermo-couples described, and have mentioned it as it was found very satisfactory. The fused bead which united the wires was then hammered out into a thin disk, and the junction mounted in the axis of the sodium tube, the wires being insulated with thin glass tubes which passed through a larger tube as shown in fig. 4.

The couple was calibrated in baths of molten lead, zinc, tin, and aluminium before and atter the experiment. The determinations at the lower temperatures and densities were made with the light from the helium tube, those at high temperatures with the green light from a mercury tube. This was necessary since a mass of very dense sodium vapour not only absorbs helium light strongly, but owing to its high dispersion makes the fringes invisible, the different wavelengths in the D line (which is of course of finite width) being shifted by different amounts. If the increase in the density with increasing temperature followed the same law as in the experiments of Jewett, the optically determined densitycurve should agree with that found by the gravimetric method employed in his work. For example, he found that the 
density at $412^{\circ}$ was six times as great as at $385^{\circ}$, and we should consequently look for a fringe-shift at the higher temperature six times as great as at the lower. This, however, was by nt means the case, the fringe-shift being only about twice as great. This is, on the whole, exactly what we should expect under the conditions of the experiment, for we must remember that our column of sodium vapour terminates in a vacuum, if the expression is allowable. If the sodium were heated in a tube provided with end plates which were not acted upon by the vapour, so that the whole could be brought to a constant temperature, it is probable that agreement with the results obtained by Jewett would be obtained. As it is, we have a cold condenser at each end of the column, and only obtain vapour of any considerable density through the circumstance that the rate of diffusion is comparatively slow.

I hope in the near future to determine the actual density under the conditions of the experiment.

The density at a given temperature was found to depend, to a large extent, upon the previous treatment of the tube. If the tube was heated for the first time to a temperature of say 350 degrees, a shift of say 10 helium fringes would be observed. If the tube was then raised to a red heat and allowed to cool, on reheating it to the same temperature $\left(350^{\circ}\right)$ a shift of perhaps 15 fringes would be found. It appeared as if the density at a given temperature was much greater if a sodium "dew" had been formed over the entire inner surface of the tube, by the condensation of the dense vapour given off at the high temperature; in other words, the density at a given temperature was proportional to the surface of molten sodium which was exposed.

The determinations were made in glass tubes for the reason that in steel tubes the transition from the heated to the cool portion of the tube was not sufficiently sudden to ensure uniform density in the cylinder of vapour. The Jena-glass tubes could, however, be raised to a full red heat by means of the coil of iron wire without showing any trace of sodium vapour in the portions protruding from the helix.

In order to obtain consistent results, it was found necessary to heat the tube very gradually in order to allow the thermoelement time to take the temperature of the vapour. One observer watched the scale of the galvanometer, the other counted the fringes as the tube was heated or cooled. The helium fringes disappeared after a shift of something over a hundred fringe-widths had occurred, but by dropping a plate of plane parallel glass into the air-path of the interferometer 


\section{Prof. R. W. Wood on the Dispersion of Sodium}

they could be made distinct again and the count continued. In this way it has been possible to record shifts as high as 400 fringe-widths. Beyond this point it is impossible to go with the helium light, the components of helium light becoming too much spread out by the dispersion of the vapour to be brought together again by going out any farther in the fringe system. To extend the observations to higher temperatures and greater vapour-density, the green radiation from a mercury-tube was used, with which fringe-shifts as high as 100 fringe-widths were observed, corresponding to a shitt of 2500 helium fringes. In this way it has been possible to obtain measures of the optical density of the rapour under the specified conditions from a temperature of $280^{\circ}$, at which point the fringes commenced to move, to above $650^{\circ}$, at which temperature the dense vapour was luminous, the entire section of the tube appearing filled with a dull red glow. Plotting the fringe-shifts as abscissa and the temperatures as ordinates, we obtain curves of form similar to the density-curve obtained by Jewett, though by no means coincident with it, for reasons which $I$ have already given.

The observations were made both when heating and cooling. At first it was found that the shift obtained on cooling was greater than the one observed during the heating; in other words, the fringe system did not return to the starting-point. This was found to be due to hydrogen gas liberated from the sodium, which, from the circumstance that it shifts the fringes in the opposite direction from the shift produced by the sodium vapour, retards the motion of the system during its liberation, and by remaining unabsorbed in the tube prevents the fringes from returning to the starting-point. This trouble was eliminated by heating the tube to a high temperature and setting the mercurial pump in operation. The observations extended over several weeks, many tubes both of steel and glass being used. The length heated was varied and the different series of observations compared. Most of this work was only useful in determining and eliminating the sources of error.

The final series, which was considered the best, was made with a tube of Jena glass, the length of the heated portion being $8 \mathrm{cms}$. The retardation, or rather the acceleration, since we are working with light on the blue side of the absorption-band, is due to a column $16 \mathrm{cms}$. long, since it is twice traversed by the interfering beam. From the data given in this table the refractive index of the vapour for either helium light or the green mercury radiation can be calculated for any temperature by means of the 
formula $n=1 \pm \frac{m \lambda}{\epsilon}$, in which $m$ is the fringe-shift and $\epsilon$ the length of the column ( 16 cms.), the + or - sign being used according to the direction of the shift.

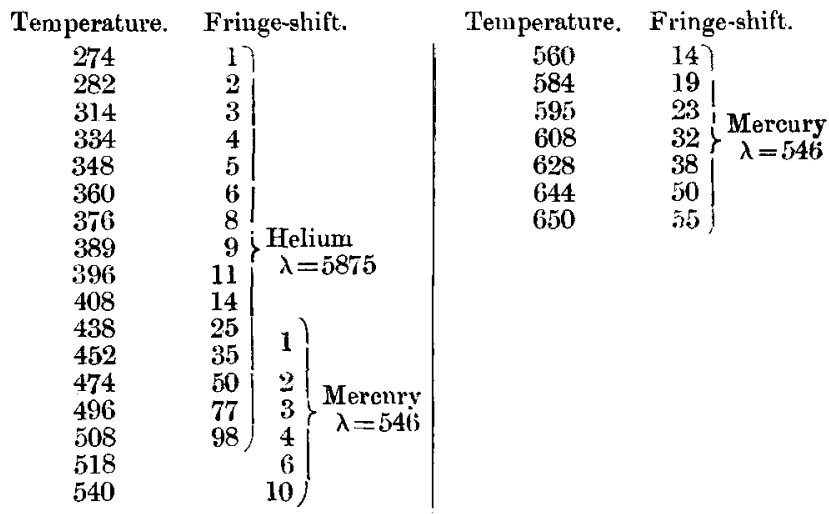

Curves are given for these optically determined densities in Plate XI., Curve 3.

\section{The Refraction and Dispersion of Sodium Vupour of Great Density.}

A knowledge of the absolute value of the refractive index of the vapour and its dispersion enables us to compile a table of the refractive indices for all wave-lengths, for vapours of varying density. This has been done for the very dense vapour obtained by heating a vacunm-tule containing the metal to a temperature of $644^{\circ} \mathrm{C}$.

A column of the vapour at this temperature $8 \mathrm{cms}$. in length examined by transmitted light has a distinct blue colour, as a result of the channelled absorption-spectrum.

The values are given in the following table, and will be spoken of in future as "observed values," to distinguish them from values calculated from the dispersion formula. It must be remembered that sodium vapour as dense as that with which we are dealing in the present case has an absorptionband at the $\mathrm{D}$ lines broad enough to completely cut out everything down to and even below the helium line, at least for all thicknesses with which it is possible to work. On this account we are obliged to calculate the refractive indices within this region from observations made with a less dense vapour, a method which in the present case is probably allowable within certain limits. A thin enough sheet of the vapour 
318 Prof. R. W. Wood on the Dispersion of Sodium

would probably transmit light within this region with a velocity indicated by the calculated indices. The question of selective reflexion at the surface and refusal to transmit the radiation will be considered presently.

In the following table the wave-lengths are given in the first column, the fringe displacements in comparison with helium light in the second column. These values, with the exception of those in the extreme red, blue-violet, and ultraviolet, were obtained with the interferometer.

In the third column are given the actual fringe-shifts which would be found for a layer of vapour $8 \mathrm{cms}$. thick (effective thickness $16 \mathrm{cms}$.), and in the fourth column the refractive indices. The indices ealculated from the dispersion formula are given in the fifth column, for the salse of comparison with the observed values. More will be said of them in the next section.

\begin{tabular}{|c|c|c|c|c|}
\hline$\lambda$. & $\begin{array}{c}\text { Relative } \\
\text { Fringe-shift. }\end{array}$ & $\begin{array}{l}\text { Total } \\
\text { Shift. }\end{array}$ & $\begin{array}{l}\text { Refractive } \\
\text { Index Obs. }\end{array}$ & $\begin{array}{c}\text { Refractive } \\
\text { Index Calcul. }\end{array}$ \\
\hline 7500 & 2 & 25 & 1.000117 & \\
\hline 6310 & 4 & 55 & 1.000197 & \\
\hline 6200 & 6 & 75 & 1.000291 & 1.000285 \\
\hline 6137 & 7 & 88 & 1.000335 & \\
\hline 6055 & 11 & 138 & 1.000523 & 1.00052 \\
\hline 6013 & 14 & 175 & 1.000658 & \\
\hline 5977 & 20 & 250 & 1.000934 & \\
\hline 5960 & 25 & 313 & $1 \cdot 001164$ & \\
\hline 5942 & 33 & 413 & $1 \cdot 001532$ & \\
\hline 5916 & 60 & 750 & $1 \cdot 002972$ & \\
\hline 5875 & 100 & 1250 & 0.995410 & •9958 \\
\hline 5867 & 67 & 834 & 996929 & -99692 \\
\hline 5858 & 50 & 625 & 997711 & \\
\hline 5850 & 40 & 500 & .998172 & .99815 \\
\hline 5843 & 33 & 413 & .998492 & \\
\hline 5827 & 25 & 313 & .998862 & \\
\hline 5807 & 20 & 250 & 999093 & \\
\hline 5750 & 11 & 138 & .999505 & \\
\hline 5700 & 9 & 113 & .999599 & \\
\hline $56 \overline{0} 0$ & $7 \cdot 4$ & 92 & 999650 & \\
\hline 5460 & 4 & 50 & .9998294 & \\
\hline 5400 & $3 \cdot 6$ & 45 & 9998481 & \\
\hline 5300 & $2 \cdot 9$ & 36 & .9998807 & 999885 \\
\hline 4500 & $1 \cdot 4$ & 17.5 & 9999508 & .999965 \\
\hline 3610 & $1 \cdot 09$ & $13 \cdot 6$ & .9999698 & \\
\hline 3270 & 9 & $11 \cdot 4$ & .9999768 & 999987 \\
\hline 2260 & 7 & $8 \cdot 7$ & 9999877 & .999995 \\
\hline
\end{tabular}

For $\lambda=\infty$ the value of $n$ is calculated to be $1 \cdot 0000275$, while for $\lambda=4167$ it is 9999975 , the deviation being the same in both cases, but in opposite directions. The complete dispersion-curve will be found on Plate XI. Curve 2, the 
Vapour in the Visible and Ultra-riolet Regions.

numbers representing the ordinates and abscissæ being outside of the large rectangle.

Refractive Indices in the vicinity of the $\mathrm{D}$ lines.

\begin{tabular}{ll}
\multicolumn{1}{c}{$\lambda}$. & \multicolumn{1}{c}{$\varkappa$ Calc } \\
5875 & $0 \cdot 9958$ \\
5882 & $0 \cdot 9890$ \\
5885 & $0 \cdot 9830$ \\
58866 & $0 \cdot 9750$ \\
58884 & $0 \cdot 9450$ \\
58896 & $0 \cdot 697$ \\
5991 & $1 \cdot 0046$ \\
5904 & $1 \cdot 0092$ \\
5901 & $1 \cdot 0138$ \\
58944 & $1 \cdot 0184$ \\
58976 & $1 \cdot 0557$ \\
5897 & $1 \cdot 094$ \\
58964 & 1.386
\end{tabular}

Application of the Results to the Dispersion Formula.

The simplest form of the dispersion formula, developed from electromagnetic considerations for a medium with a single absorption-band, is

$$
n=1+\frac{m \lambda^{2}}{\lambda^{2}-\lambda_{m}^{2}}
$$

in which $m$ is a constant, $\lambda$ the wave-length of the light employed, and $\lambda_{n}$ the wave-length at the centre of the absorptionband.

The vapour of sodium has, of course, a pair of very close absorption-bands (the D lines) which are chiefly effective in modifying the refractivity of the medium. As I have said, the ultra-violet bands affect the dispersion in their immediate vicinity, but their effect can be neglected in comparison with the stronger band, except for wave-lengths very close to them.

The first question to consider is whether we are justified in considering the $\mathrm{D}$ lines as a single absorption-band in the case of the very dense vapour. If we consider the medium as having a single band, and assign to $\lambda_{m}$ the value 5893 (a point midway between the $D$ lines), we shall find that the observed and calculated values of the refractive index agree very closely, up to within a distance of the band about equal to the distance of the $\mathrm{D}_{3}$ line of helium. If we attempt to go closer than this, we immediately find discrepancies, which become larger as we approach the $\mathrm{D}$ lines. 
The constant $m$ in the above formula can be determined from a single observation of the refractivity. It was determined from two values, namely, the refractivity for the green line of mercury $(\lambda=546)$ and that for $\lambda=585$, the latter wavelength being quite close to the $D$ lines. Since the refractivity has widely different values for these two wave-lengths, we should expect the difference between the two calculated values to be a maximum in this case, in the event of the dispersion being incorrectly represented by the formula. The values for $m$ found in the two cases were 0.000056 and 0.000054 , a surprisingly close agreement. The menn value $m=0.00055$ was taken, and the indices for a number of wave-lengths calculated. Some of these values are given in the table of refractive indices, and they will be found to agree very closely with the observed values. The values calculated between the belium line and the $\mathrm{D}$ lines are given in the second part of the table.

It will be noticed that the greatest discrepancies are found in the ultra-violet, the observed values indicating a greater deviation (negative since the index is less than unity) than we should expect theoretically. In other words, we should expect the deviated spectrum to be more nearly in coincidence with the undeviated than was found in the photographs taken through the slotted slide. I am inclined to attribute the discrepancy to the presence of hydrogen in the tube. The dispersion-tube was exhausted and sealed off from the pump before introduction into the combustion-furnace. A small amount of hydrogen is always liberated from the sodium as scon as it is heated, and without doubt the tube contained a small amount of this gas during the experiment. At the time it was not expected that this would give any trouble, but observations appear to indicate the contrary. A tube containing air or any ordinary gas acts like a prism with its thin edge down, when the floor of the tube is strongly heated. This is owing to the rarefaction of the gas along the heated floor. Now the sodium prism is turned in exactly the opposite way, the denser portions being close to the floor. But on the blue side of the sodium absorption-band the index of refraction is less than unity; consequently the oppositely oriented prisms (hydrogen and sodium vapour) deviate in the same direction, and the effect of the bydrogen prism will be greatest in the ultra-violet for obvious reasons. The result will be that slightly too great deviations will be found in this region, which is precisely what we require to explain the discrepancies between the observed and the calculated values. 
Unfortunately sufficient time did not remain for the repetition of the experiment with a tube permanently in connexion with the pump, but the omission is not serious, for I feel certain that the explanation given above is the correct one.

An inspection of the formula shows us that, according as we are on the red or blue side of the absorption-band, the refractive index is given by adding to or subtracting from unity the value of the constant $m=0 \cdot 000055$ multiplied by the fraction $\frac{\lambda^{2}}{\lambda^{2}-\lambda_{m}^{2}}$. In the case of all other substances showing anomalous dispersion, aniline dyes for example, to the dispersion of which the formula has been applied, the value of this fraction never exceeds 3 owing to the impossibility of applying it to wave-lengths very close to the centre of the band. For example, in the case of the dispersion of nitroso-dimethyl-aniline, with its strong absorption-band at $\lambda=43$, we cannot obtain accurate data nearer than $\lambda=50$. In this case $\frac{\lambda^{2}}{\lambda^{2}-\lambda_{m}^{2}}=3.9$.

In the case of sodium vapour the value of the fraction may be several hundred or even thousand. In the case of $\lambda=5882$ the fraction is 367 , and yet the observed and calculated values agree closely. For $\lambda=58884$ the fraction is 1940 , and for $\lambda=5889 \cdot 6$ we have a value as high as $3: 44$. The product of these very large numbers and the small fraction 0.000054 give, however, values of the index which are in close agreement with the observed values.

Discrepancies occur in the immediate vicinity of the $\mathbf{D}$ lines which can be explained in the following way. To get values in any way consistent with the observed values, it was necessary to assign to $\lambda_{m}$ the value of the $\mathrm{D}_{2}$ line, the mean value 5893 being too far removed from the wave-lengths in question to give the requisite steepness to the curve. The calculated values, therefore, apply to a medium with a single band at $D_{2}$, and with a constant $m=0 \cdot 00055$. This gives us a pretty good approximation to the observed curve, but the latter is due to the combined effects of the bands $D_{1}$ and $D_{2}$, the presence of the $D_{1}$ band tending to make the observed curve flatter than the calculated. A more correct approximation could be obtained by assigning to $\lambda_{m}$ a value intermediate between $\mathrm{D}_{2}$ and 5893. The proper method of procedure would of course be to make use of two members in the dispersion formula, one for $D_{1}$ and the other for $D_{2}$. Thus

$$
n^{2}=1+\frac{m \lambda^{2}}{\lambda^{2}-\lambda_{m}^{2}} \times \frac{m^{\prime} \lambda^{2}}{\lambda^{2}-\lambda_{m^{i}}^{2}} .
$$




\section{Prof. R. W. Wood on the Dispersion of Sodium}

If $m$ and $m^{\prime}$ were each assigned the same value obtained by dividing our original value by 2 , in all probability a very close approximation would be obtained in the region in question. This has not been done for two reasons. In the first place, it does not appear as if much would be learned by the procedure; and in the second place, $m$ and $m^{\prime}$ are not equal, as is shown by the stronger dispersion near $D_{2}$, and until the relative values have been determined we are not in a position to write the two-member formula accurately. It is doubtful whether anything new would come out of such a determination, and it was. on that account not attempted.

Another matter of considerable interest is the question of the indices represented by the square root of a negative quantity in the immediate vicinity of the absorption-band on the blue side. Lord Kelvin interprets this as indicating that no light of such wave-lengths enters the medium; in other words, it is metallically reffected. It is in this way that he has explained the apparent greater brondening of the $\mathrm{D}$ lines on the more refrangible side in some of Becquerel's photographs. In the case with which we are dealing, the second term of our original formula does not become less than unity until we reach wave-length 58898 , which we get by equating $\frac{m \lambda^{2}}{\lambda^{2}-\lambda_{m}^{2}}$ to unity and solving for $\lambda$.

This shows us that, even with a vapour so dense that both $\mathrm{D}$ lines run together and broaden out into a wide band, we do not get values of the $e_{o}$ index which are imaginary until we are within 0.2 of an Angström unit of the D line, or, in other words, until we are within a distance of $D_{2}$ equal to $1 / 30$ of the distance between $D_{1}$ and $D_{2}$. In the case of the comparatively rare vapour employed by Becquerel, we should have to approach much closer than this to get the imaginary values. This makes it appear certain that the greater broadening on the more refrangible side, if it exists, must be assigned to some other cause than imaginary values of the refractive index.

The medium is exceptionally interesting in that its dispersion can be represented throughout the entire range of wave-lengths without taking the extinction coefficient into account, as is always necessary in the case of solids and liquids when in the vicinity of the absorption-band.

\section{I he Question of the Selective Reflexion of Sodium Vapour.}

The vapour of sodium should exhibit strong selective reflexion in the vicinity of the D lines, just as the aniline dyes 
have metallic reflecting-power for light of certain wavelengths. For obvious reasons the vapour must be very dense if the phenomenon is to be detected experimentally. It must, moreover, terminate abruptly in a flat surface. This can only be accomplished by confining it in closed vessels made of some transparent material, since in a vacuum-tube we have a gradual transition from dense to rare vapour at the free surface. I feel justified in speaking of the free surface of a gas in a vacuum-tube in this particular case. A more precise definition of the apparent surface may be an isothermal surface, on one side of which we have sodium vapour and on the other sodium fog. From such a surface we should hardly expect to get any trace of reflexion, which requires that the transition from dense to rare be sudden. If we confine the vapour in glass bulbs, we at once encounter many difficulties. The glass is at once attacked and discoloured, and we have the reflexion from the glass surfaces. An attempt was, however, made to observe the phenomenon in the following way. A small amount of the metal was sealed up in a hard glass bulb, highly exhausted. The bulb was mounted in a small air-bath of sheet-iron which could be rapidly raised to a red heat.

The reflexion of the filament of at Nernst lamp by the inner and outer surfaces of the bulb was observed through a. large direct-vision prism. The two minute points of light were seen drawn out into spectra, and it was hoped that any trace of selective reflexion in the vicinity of the $\mathrm{D}$ lines would manifest itself by a change in the relative intensities of the two spectra at the point in question.

The bulb was heated very rapidly, but no conclusive observation made. The glass discoloured so rapidly that the densest rapour could not be studied. In one case it was thought that a slight brightening in the yellow occurred, and the subject will be further investigated. Possibly by employing light polarized in such a plane as to be refused reflexion by glass surfaces, we may be able to get the desired results.

Bulbs of fused quartz become discoloured in the same way, but I have been told that fused alumina remains transparent. If it be found possible to blow a small bulb of alumina on the end of a quartz tube, the question of the selective reflexion can be easily settled.

The phenomenon is doubtless connected intimately with the fluorescence of the vapour. In studying this phenomenon with very dense vapours, I was forced to the conclusion that the illumination of the vapour with light of the wave-length 


\section{Prof. Wood on the Achromatization of Monochromatic}

of the $\mathrm{D}$ lines did not provoke the fluorescence. Wiedemann and Schmidt observed a bright band in the fluoreseence spectrum in the case of vapour confined in glass bulbs which appeared to coincide with the $\mathrm{D}$ lines. The same appearance I afterwards observed independently, but on continuing the study of the subject was forced to refer the existence of this band in the yellow to sodium in the flame which heated the bulb. This I bave since found was a mistake, for on repeating the experiments with exhausted glass bulbs, I have succeeded in stirring up a faint fluorescence with approximately monochromatic light from the illuminator already referred to, of wave-length equal to that of the $\mathrm{D}$ lines. The failure to observe it in the case of the experiments made last year by Mr. Moore and myself was due to the fact that this yellow light was removed from the incident beam by the sodium vapour before the light-rays met at the focus. This work is still in progress, and the fluorescent spectra given by the vapour when illuminated with monochromatic light of various wave-lengths have been photographed.

The investigations recorded in the present paper have been made possible by a very generous grant from the Rumford Fund, and I wish to express to the Trustees of the fund my appreciation of and thanks for the aid which $I$ have received.

I wish also to express my appreciation of the very faithful work done by my assistant, Mr. A. H. Pfund, who has worked with me and made many valuable suggestions, and my thanks to the Board of Trustees of the Carnegie Institution for the means placed at my disposal by which I have been able to secure his services.

Johns Hopkins University, Baltimore, MId, May 28, 1904 .

XXXIII. The Achromatization of Approximately Monochromatic Interference Fringes by a Highly Dispersive Medium, and the conseguent Increase in the Allowable Path-difference. By R. W. Wood, Professor of Experimental Physics, Johns Hopkins University*.

THE results recorded in the present paper were, for the most part, obtained during the progress of an investigation of the dispersion of sodium vapour. As I have mentioned in the previous paper, the path-difference under

* Communicated by the Author. 
Phil. Mag. Ser. 6, Vol. 8, Pl. X.

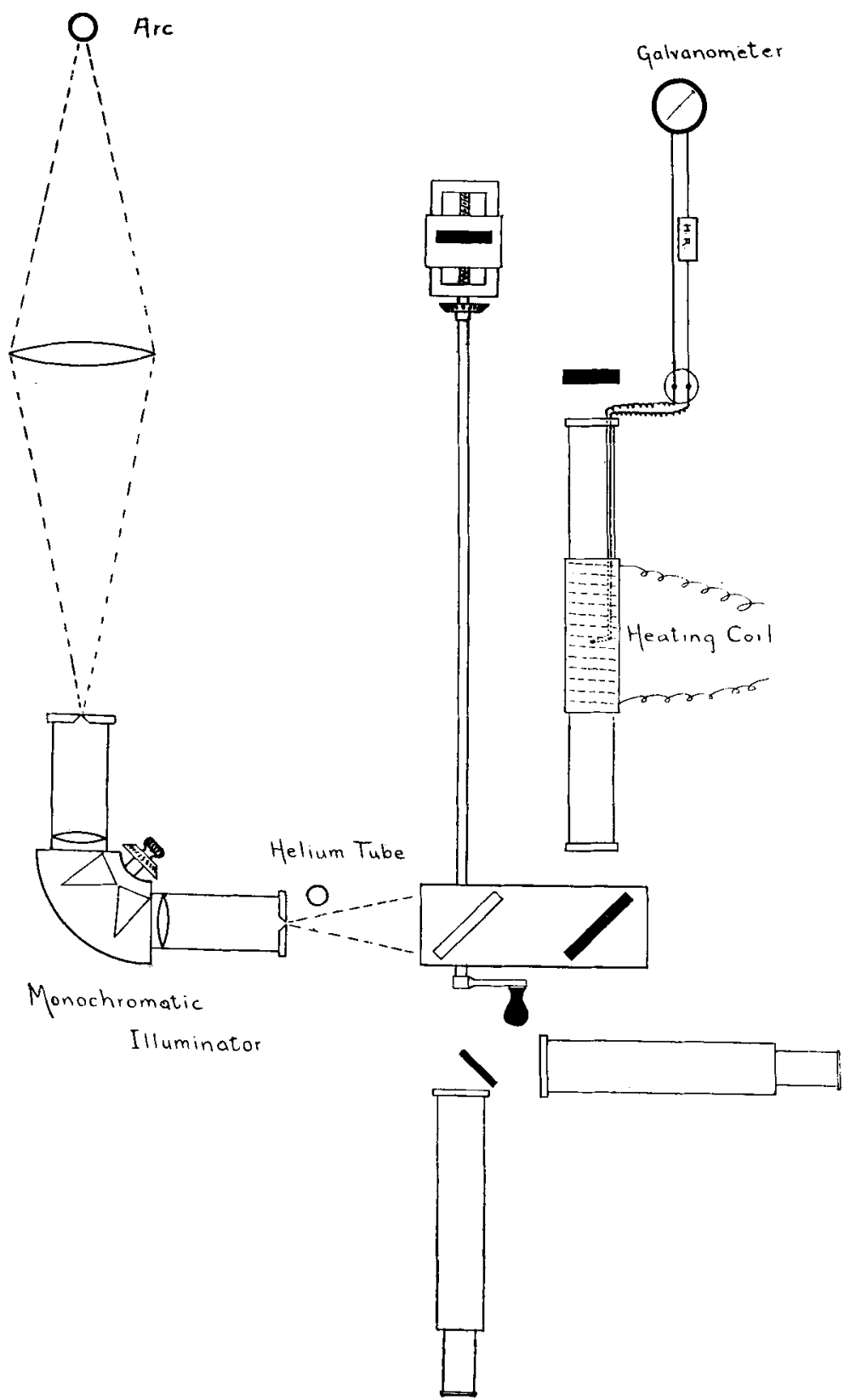


Phil. Mag. Ser. 6. Vol. 8. P1. XI.

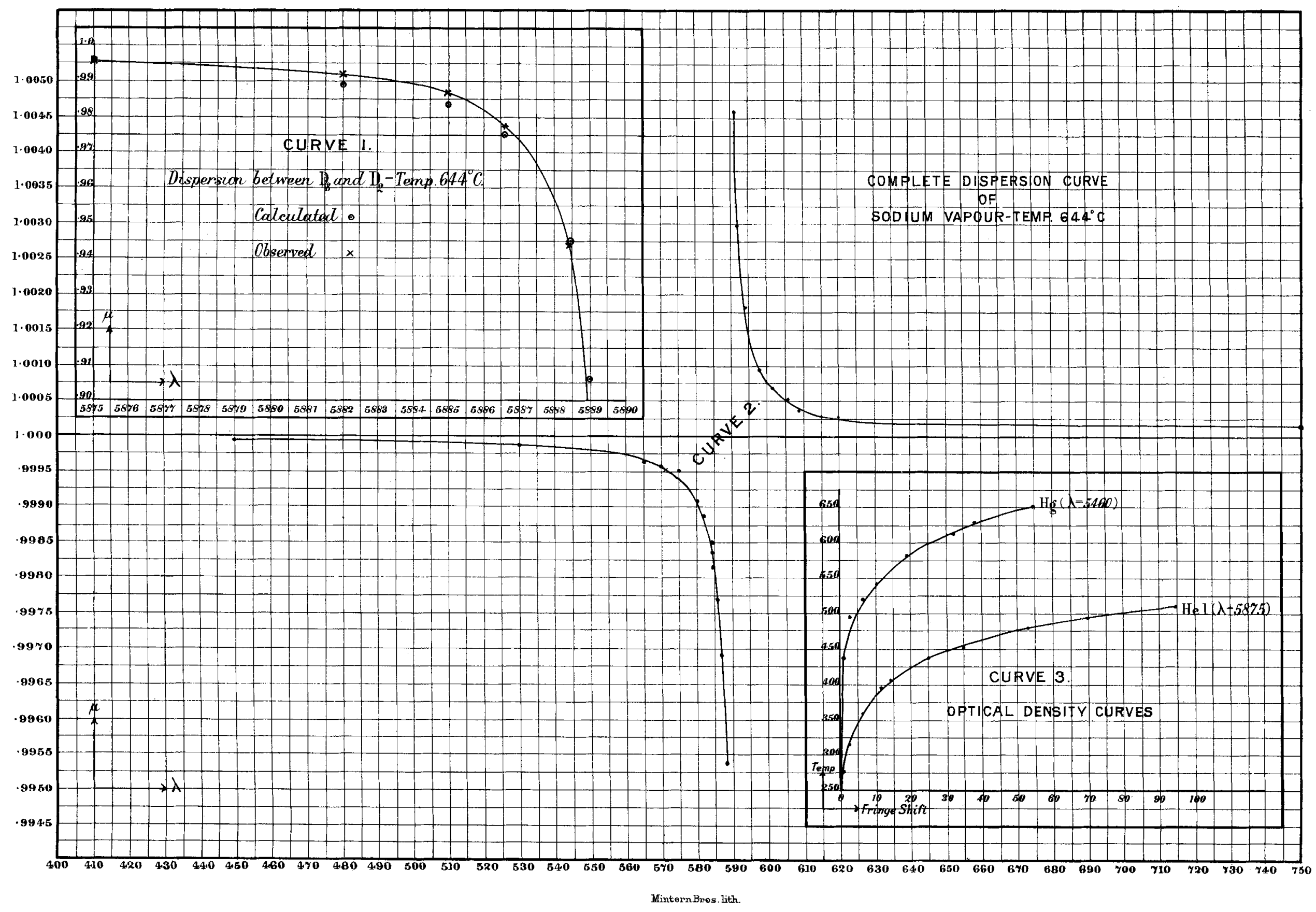


Phil. Mag. Ser. 6, Vol. 8, PI. XII.
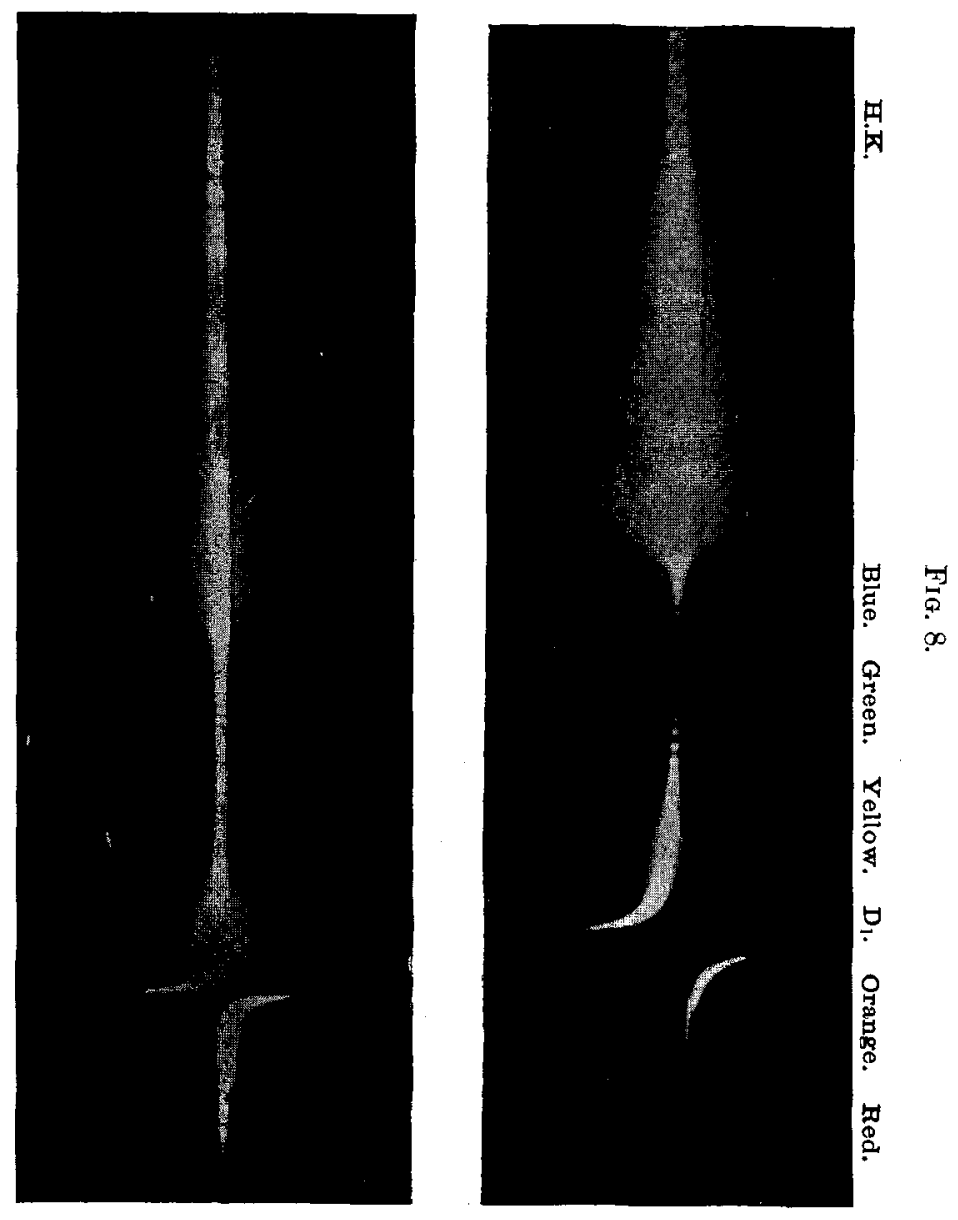